\title{
Demonstrative educational haptic manipulator robot: A teaching aid in Mechatronics
}

\author{
Afonso Pereira \\ up201505870@fe.up.pt \\ Faculty of Engineering, University of Porto, Rua Dr. \\ Roberto Frias, 4200-465 Porto \\ Porto, Portugal \\ José Gonçalves \\ goncalves@ipb.pt \\ Research Centre in Digitalization and Intelligent Robotics \\ (CeDRI), Instituto Politécnico de Bragança, Campus Santa \\ Apolónia, 5300-253 \\ Bragança, Portugal
}

\begin{abstract}
In this paper it is presented an educational experiment, that consists of a mechatronic system applied to demonstrate concepts such as prototyping, control, haptic feedback and the use of different sensors and actuators. The described mechatronic system is based on the use of two identical manipulators, being physical devices commonly used in the industry. The physical components of the manipulators were 3D printed, being the original model of each manipulator the "EEZYBotArm MK2". This, already existent prototype was modified, with the necessary changes, to fulfill the requisites of the proposed system, being included load cells to provide measurement of the applied forces, and the robot gripper was also modified, being applied an electromagnetic actuator.
\end{abstract}

\section{CCS CONCEPTS}

- Robotics Education;

\section{KEYWORDS}

Manipulator, Prototyping, Sensor, Actuator, Haptic Feedback

ACM Reference Format:

Afonso Pereira, Vítor H. Pinto, José Gonçalves, and Paulo Costa. 2021. Demonstrative educational haptic manipulator robot: A teaching aid in Mechatronics. In Ninth International Conference on Technological Ecosystems for Enhancing Multiculturality (TEEM'21) (TEEM'21), October 26-29, 2021, Barcelona, Spain. ACM, New York, NY, USA, 8 pages. https://doi.org/10.1145/ 3486011.3486415

\section{INTRODUCTION}

Mechatronics is a multidisciplinary subject that deals with many concepts such as Mechanics, Electronics, Control and Computer

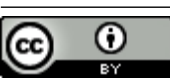

This work is licensed under a Creative Commons Attribution International 4.0 License.

TEEM'21, October 26-29, 2021, Barcelona, Spain (C) 2021 Association for Computing Machinery. ACM ISBN 978-1-4503-9066-8/21/10.

https://doi.org/10.1145/3486011.3486415

\author{
Vítor H. Pinto \\ vitorpinto@fe.up.pt \\ Faculty of Engineering, University of Porto, Rua Dr. \\ Roberto Frias, 4200-465 Porto \\ Porto, Portugal \\ Paulo Costa \\ paco@fe.up.pt \\ Faculty of Engineering, University of Porto, Rua Dr. \\ Roberto Frias, 4200-465 Porto \\ Porto, Portugal
}

Science $[1,3,6,9,10]$. It is very important to have experimental kits in the classroom, both for training as well as for demonstration. In this paper it is described an educational experiment that consists of a mechatronic system applied to demonstrate concepts such as prototyping, control, haptic feedback and the use of different sensors and actuators.

Nowadays, robotic manipulators are vastly used in diverse areas such as goods production, medical surgeries and handling of dangerous materials $[2,4,5,8]$. However, in order for the manipulator to be useful, it is first needed to program it to do a required task. This process of "teaching" the robotic arm becomes a more accessible procedure if it is possible to replicate the desired movements manually. To do this, it is possible to make use of remote control which provides the possibility to control a manipulator from a distance. Nonetheless, for this type of control to be effective and accessible it is important that the user acquires a good knowledge of the remote environment. This is usually done with the help of cameras and microphones which provide image and sound feedback. The proposed prototype aims to enhance this type of remote control for teaching robotic manipulators with the addition of a haptic system. This provides the user with a tactile sensation of the remote environment and thus a better control of the robotic arm. The developed system is composed of two robotic manipulators with three degrees of freedom each, which were modified to contain force sensors, and two graphical interfaces we developed, that allow a better control of the robotic arms. It is possible to record sequences of positions captured using the application as well as repeat those sequences. Each manipulator is composed of three servo motors, a micro-controller, three force sensors and one electromagnet that serves as the tool. The control scheme expected for the local manipulator is compliance control, which is when the user manually moves the manipulator to the required positions by applying forces on the arm itself. Like any remote system, when the local robotic manipulator changes poses, the remote manipulator should mimic that pose, as demonstrated in Figure 1.

The type of haptic feedback, chosen for this system, was force feedback which will be made possible using the servo motors on the local manipulator. This will be useful in situations where the 

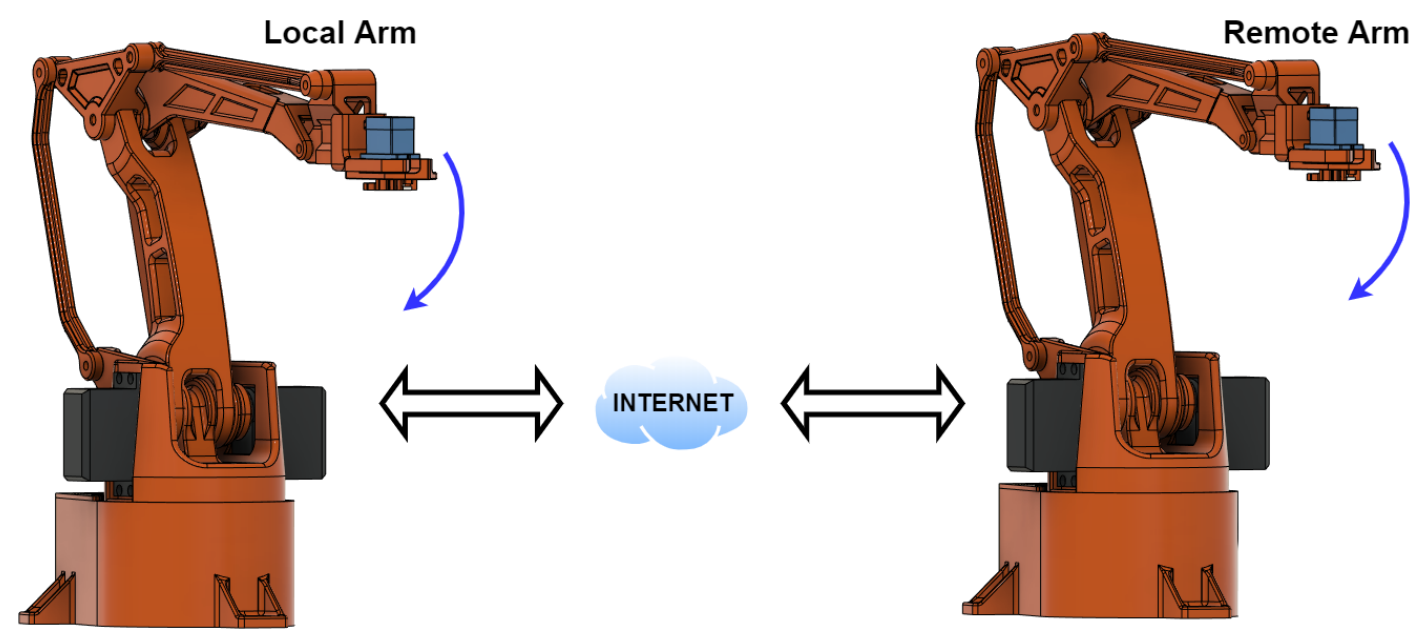

Figure 1: System concept diagram.

remote manipulator collides with something in it's environment giving the user better sense.

The paper is structured as follows, initially the system architecture is described, then the manipulator prototyping is described, then the haptic feedback results are described and finally some conclusions and future work are presented.

\section{SYSTEM ARCHITECTURE}

The system architecture used for this project is presented in Figure 2. The system is composed of two main subsystems: the local subsystem and the remote subsystem.

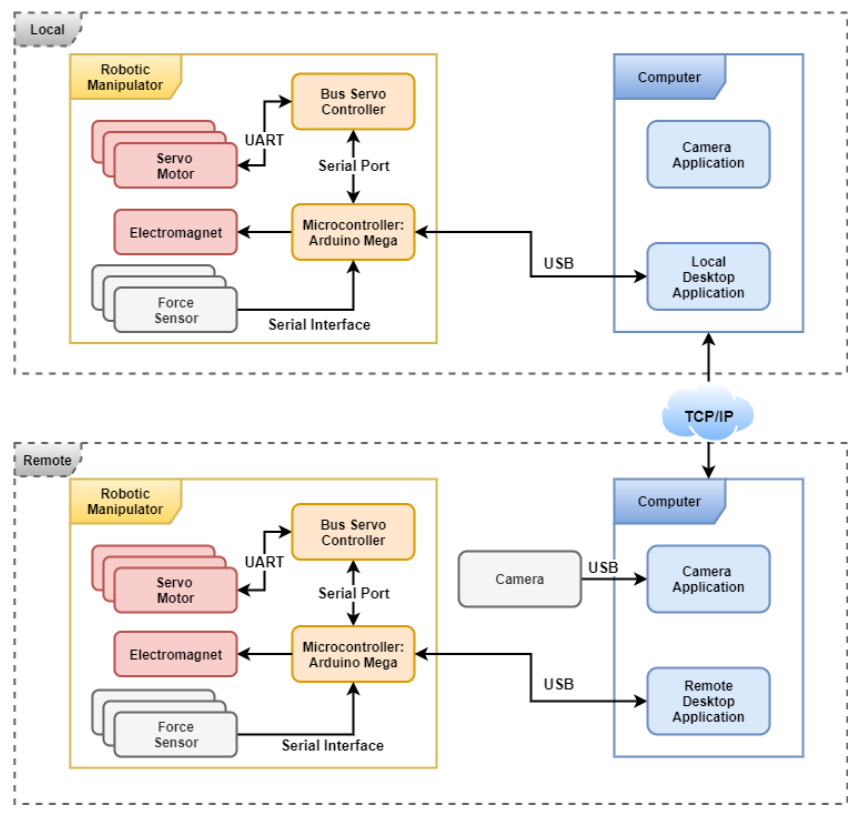

Figure 2: System Architecture.
Both subsystems are very similar to one another in terms of components with the exception of a camera sensor which is used in the remote subsystem and not in the local one. This camera is connected via USB and is used to provide visual feedback of the remote environment to the local system and thus give a better experience to the user operating the system.

Each subsystem along with the relations between them will be explained in the following subsections.

\subsection{Manipulator Subsystem}

The robotic manipulator subsystem consists of:

- One Arduino Mega 2560;

- One Bus Servo Controller;

- Three LX16-A Servo Motors;

- One Electromagnet;

- Three Force Sensors:

- One Load Cell;

- One HX711 ADC.

Each component relates to one another as shown in Figure 3. Figure 4 demonstrates a diagram with the connections made to the Arduino Mega.

The Arduino Mega is the brain of the manipulator. It is in charge of operating the actuators, the electromagnet and the servo motors, and of reading the sensors, the load cells. Furthermore, it is also responsible for executing the control loop of the robotic arm and is applied to communicate all this information with the computer.

The microcontroller reads force measures from the load cells with the help of the HX711 ADC at a frequency of $80 \mathrm{~Hz}$ which could be a limitation. However, because the control loop is set to execute every 40 milliseconds, which is $25 \mathrm{~Hz}$, this is not a problem since it reads only 1 measure for each cell every control loop.

The electromagnet requires a control signal to operate. This control signal is sent by the microcontroller and is a simple ON/OFF type control. Finally to control the servo motors, a controller board is used which interfaces with the Arduino Mega via serial port. 


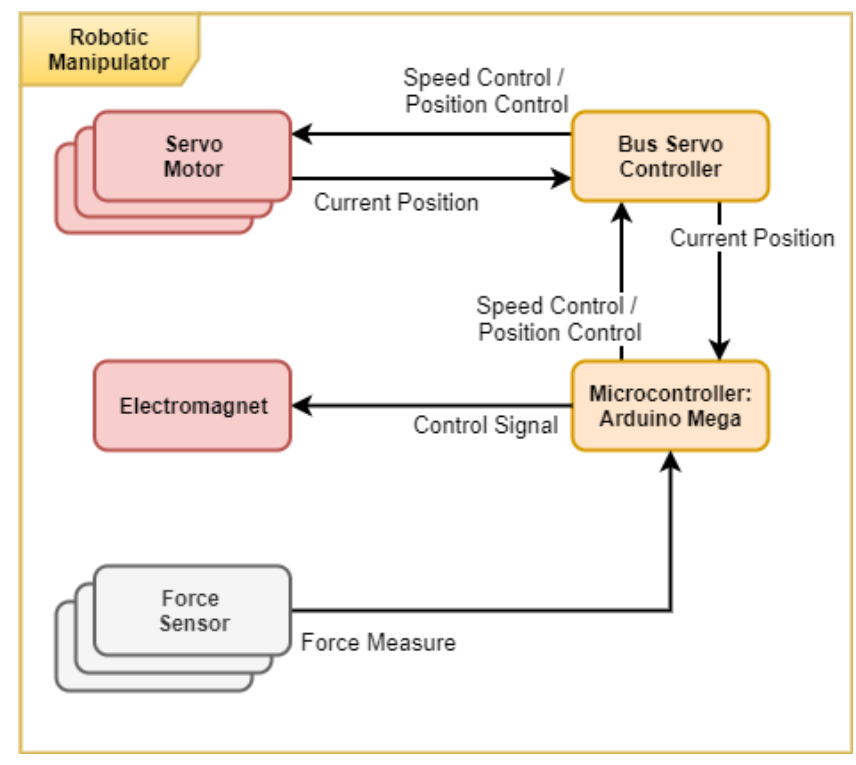

Figure 3: Manipulator Architecture.

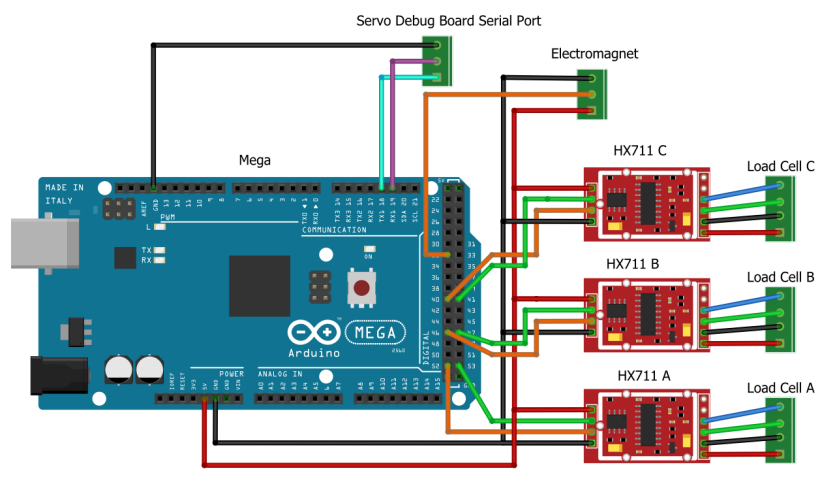

Figure 4: Bread Board Circuit Diagram.

\subsection{Manipulator to Computer Connection}

As mentioned before the microcontroller is responsible for the communication between the manipulator and the computer application. This is done using a USB cable that connects the Arduino Mega to a computer port and thus a serial communication is created.

The information passed from one subsystem to the other is practically the same for both the local and remote systems, as seen in Figure ??. In both systems the microcontroller sends information about the servos and the load cells along with the current state of the electromagnet (either active or not). Moreover, the state of the Arduino Mega and the time it took to execute the last control loop is also sent as a way to help debug the microcontroller.

Also in both systems, the desktop application sends commands to the microcontroller for position control and to operate the electromagnet. However, in the local system, the desktop application also sends the values of the remote load cells which is then used by the microcontroller to implement the haptic feedback.

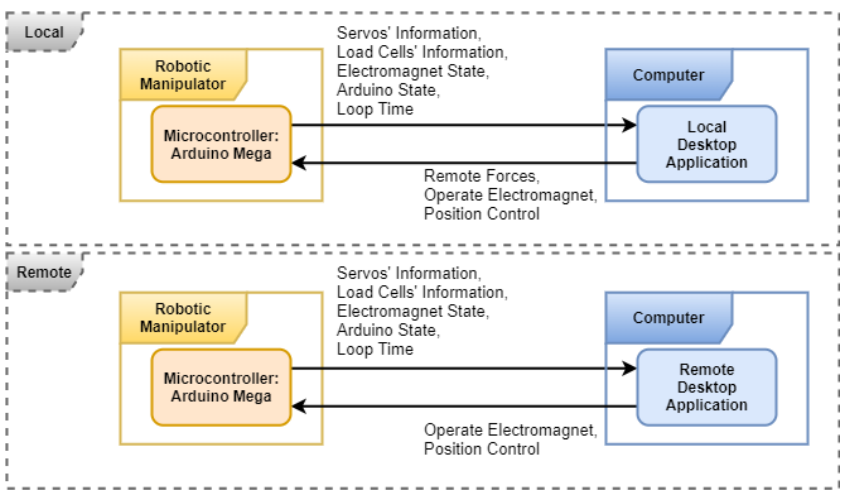

Figure 5: Manipulator to Computer Architecture.
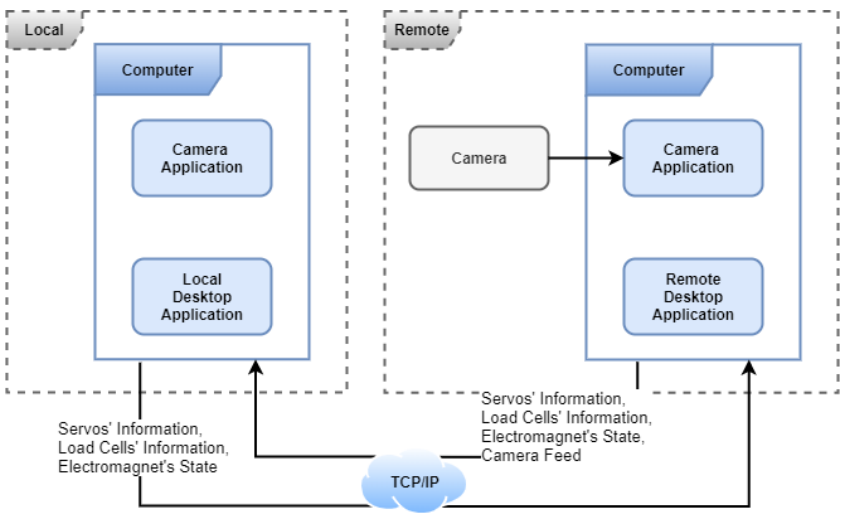

Figure 6: Computer to Computer Architecture.

\subsection{Computer to Computer Connection}

Finally, the relation between both computer subsystems is done via Transmission Control Protocol (TCP)/ Internet Protocol (IP), Figure 6.

The information passed over this medium from both subsystems consists of the information about the servos and the force sensor's measures along with the electromagnet's state. Apart from this information, the remote subsystem also sends data to the local subsystem about the camera feed it is capturing, which is then displayed by in an application.

\section{MANIPULATOR PROTOTYPING}

The manipulator was prototyped using $3 \mathrm{D}$ printing technology. This prototyping method is nowadays a very accessible way to create objects from a digital 3D model. The technique used was Fused Deposition Modeling (FDM) and the material used for printing each part was white PolyLactic Acid (PLA). The models are represented in orange, as a way to contrast with the white background of the document. The complete printed and assembled robotic arm can be seen in Figure 7. Details of each modification, from the original to the final prototype, will be detailed in the next subsections. 


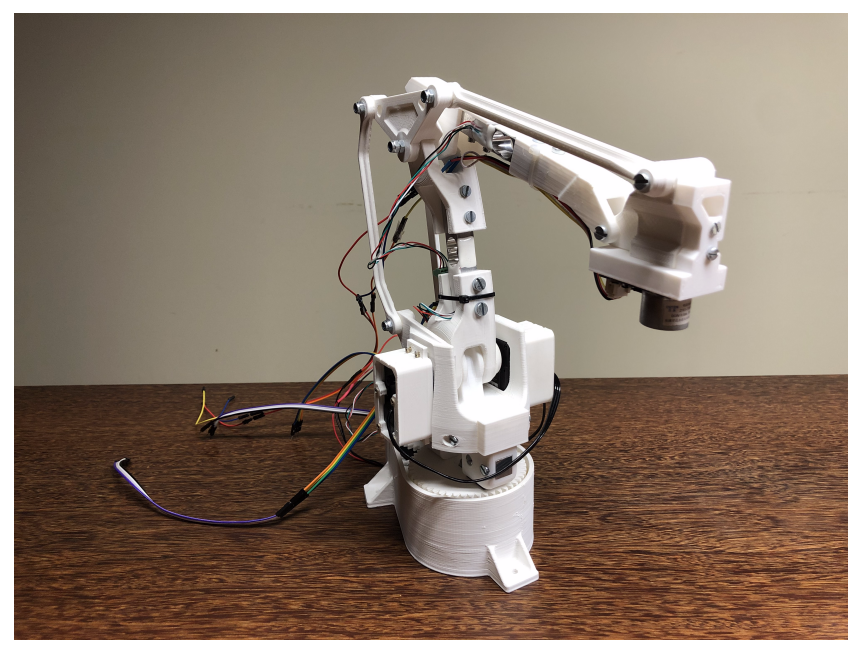

Figure 7: Picture of Printed Robotic Arm.
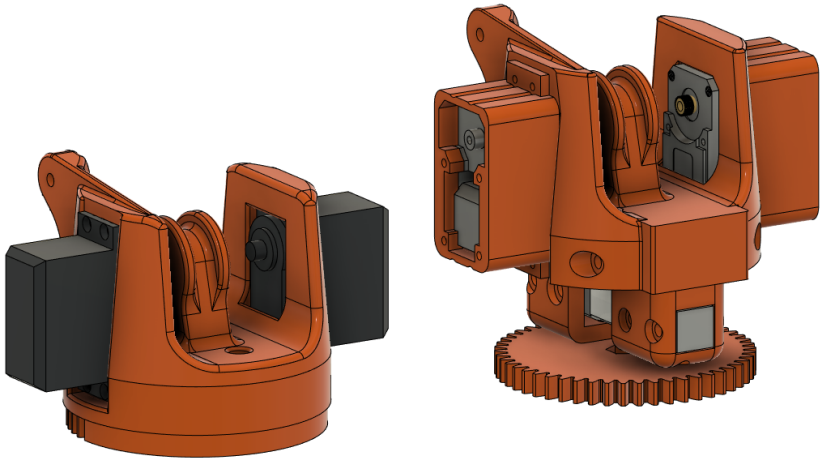

Figure 8: Before and after model of the Base.

\subsection{Body}

The body of the manipulator is a modified version of the EEZYbotArm Mk2 from daGHIZmo [7], which is a Palletizer configuration that has three DOF. The Computer Aided Design (CAD) files for this robot arm can be downloaded from the Onshape website where the author has provided them for free.This is extremely useful for the changes that need to be made to the several parts because it allows for the use of a software capable of reading and modifying these files. These modifications were made using Autodesk's Fusion 360 program.

The base of this model was original made to use servos like the MG955 or MG946R. However, because the motors used are different and don't have the same dimensions, the base of the body had to be slightly changed. Moreover, in order to measure the force applied to the base's axis of rotation, a load cell sensor had to be inserted somewhere and thus some further modifications were made. This was the case for the second and third links as well.

3.1.1 Base. The customization of the base consists of two different modifications from the original model. Figure 8 shows the original version and the modified version for comparison purposes.

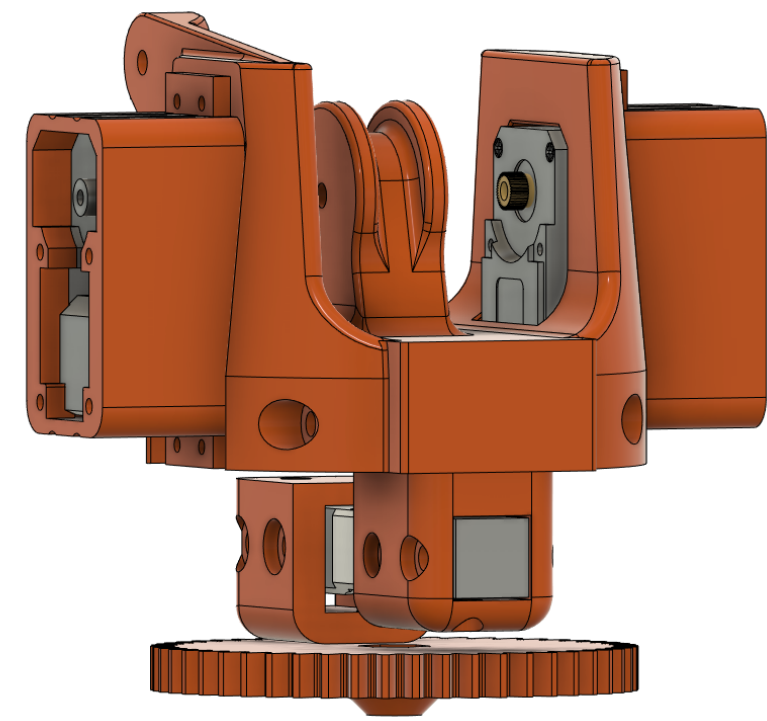

Figure 9: Model of the Base.

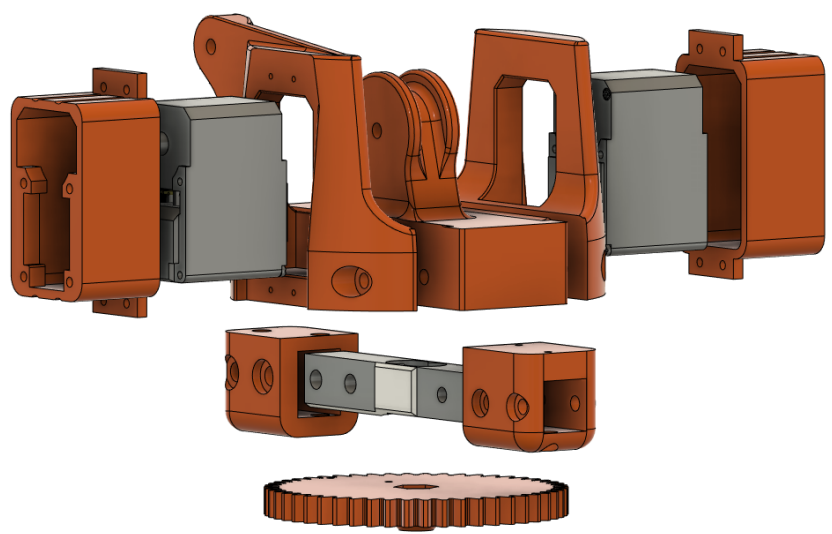

Figure 10: Exploded model of the Base.

First, the slots for the servos were changed and a holder to fix them in place was modeled. Second, a holder for the strain gauge cell was implemented. Figure 9 demonstrates the complete modified version of the base and Figure 10 demonstrates its exploded axonometric.

The servo holders are a simple casing that attaches to the backside of the servo using screws and then is attached to the base and also to the links 2 and 3 . Figure 11 shows the servo encased in its holder.

In order to implement these servo holders, there was the need to modify the base itself as well which was done to maintain the position of the axis of the old base to avoid further changes on the links.

The load cell sensor measures a force applied to it in one axis. In order for this component to work properly, both sides cannot be 


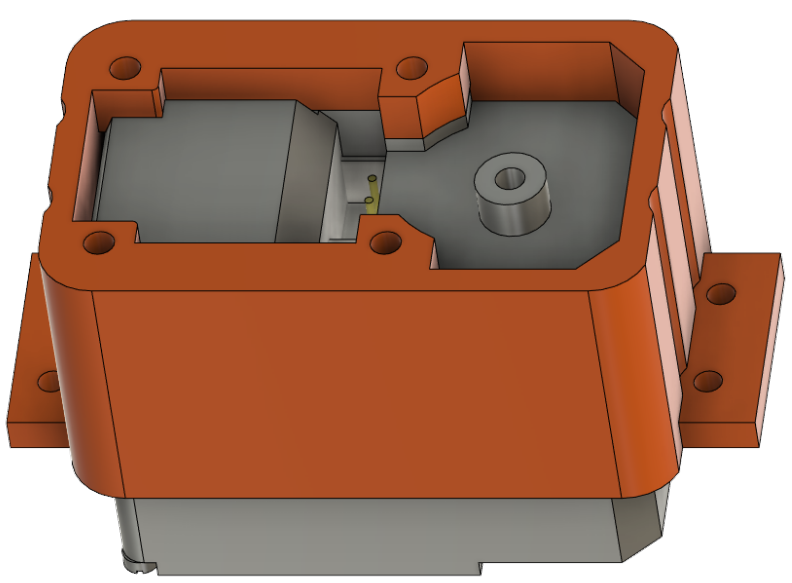

Figure 11: Model of the Servo Holder for the Base.

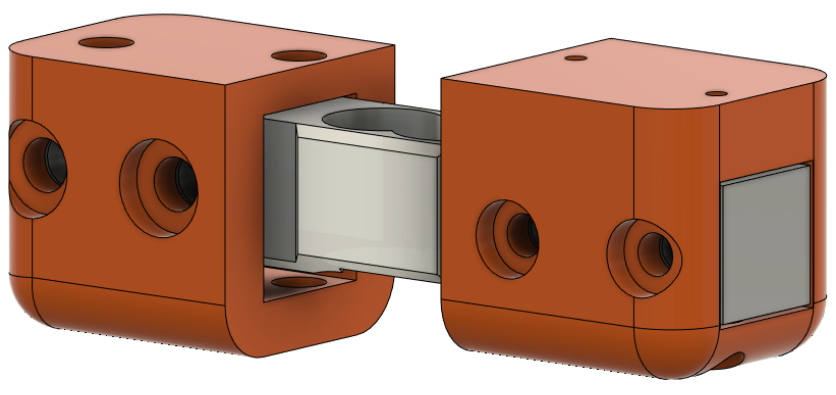

Figure 12: Model of the Cell Holder for the Base.

fixed to the same rigid body. This would prevent the sensor from flexing and thus measuring the force properly would be impossible.

The sensor used in this component was the TAL220 and Figure 12 demonstrates the holder created for it. It is composed of two separate pieces that are fixed to either the base or the full gear. This way it is possible to read the force applied to the $\mathrm{z}$-axis of rotation of the base.

3.1.2 Link 2. The changes made on the second link were simple and straightforward. The main necessity was to insert the force sensor, and for it to work the model had to be split into two separate pieces. The sensor used was the same model as the one used previously in the base, the TAL220. Apart from that, the connection between the link and the motor had to be slightly changed because of the different motors used. Both the original and modified versions of this link can be seen in Figure 13.

3.1.3 Link 3. In terms of link 3, the only change needed was the insertion of the force sensor. Similarly to link 2 , the model was split into to two separate pieces in order to place the sensor in between them. Like the previous two modifications, the sensor used in this component was the TAL220. Figure 14 shows the original model alongside the modified version. Because the motor is not directly
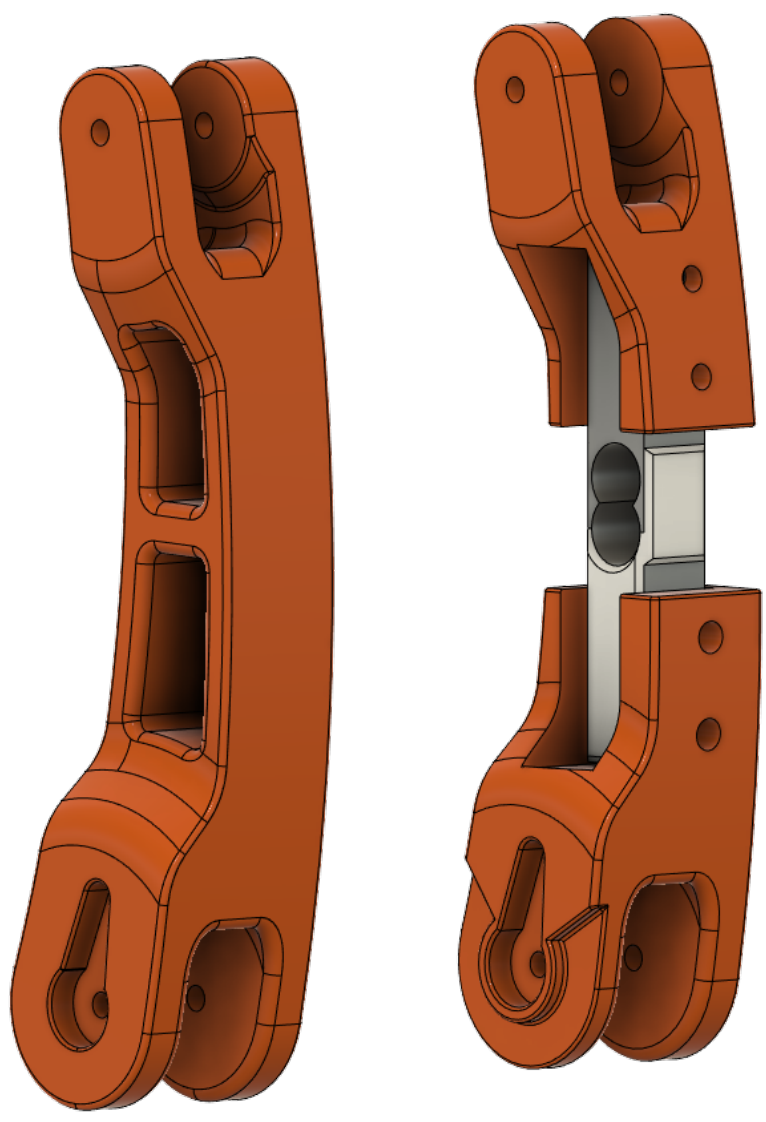

Figure 13: Before and After of Link 2.

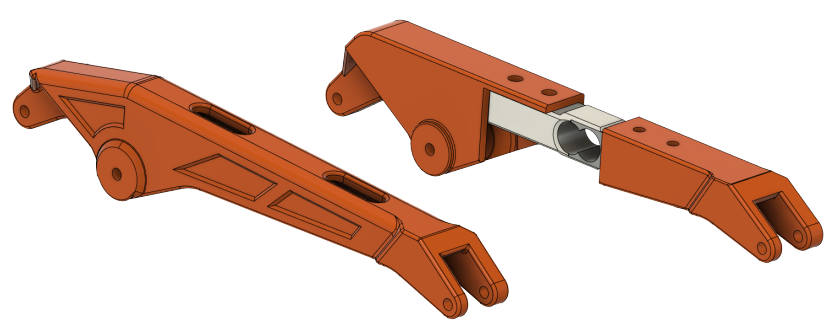

Figure 14: Before and After of Link 3.

connected to the joint, there was no need to change this model any further. However, the piece that is connected to the motor's axis had to be modified in the same manner as link 2, as shown in Figure 15. 

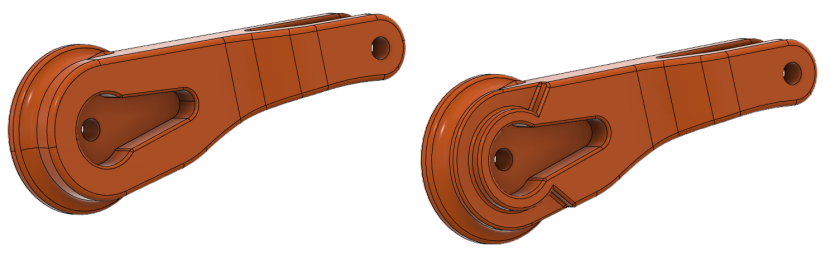

Figure 15: Link 3 Motor Connector Before and After.

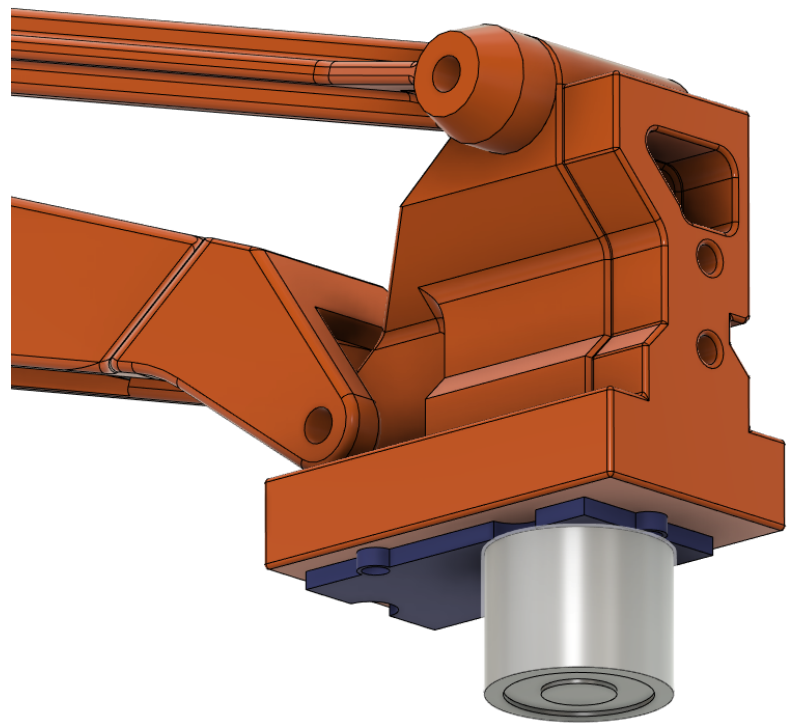

Figure 16: End Effector

\subsection{End Effector}

The end effector was changed from the original claw to an electromagnet. To do this a support that combines the electromagnet with the rest of the body was created. This was a simple piece that is demonstrated on Figure 16. This piece was developed with the intention of pointing the electromagnet downwards towards the floor instead of forwards in order to facilitate the action of picking up objects.

\subsection{Complete Manipulator}

Finally, after every modification made to the several more critical parts, it is possible to assemble the final version of the custom manipulator.

This model is presented in Figure 17 alongside the original model for comparison purposes.

\subsection{LX-16A Servo Motor}

The motors used in this project are electric of type Servo. The model chosen was the LX-16A servo from LewanSoul. This product is comprised of the motor, a servo drive, a serial bus communication interface and a potentiometer. It allows for 2 modes, continuous

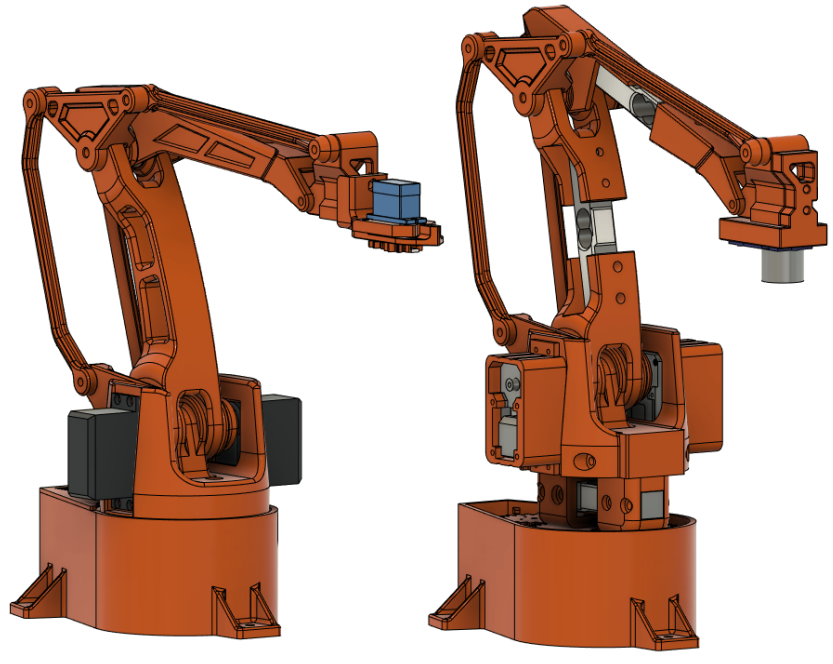

Figure 17: Original model and Modified model.

rotation or position control. Some specifications for this model are shown in Table 1. Furthermore, the servo includes a Light Emitting Diode (LED) that is used to signal possible operation errors.

\begin{tabular}{|c|c|}
\hline Net Weight & $52 \mathrm{~g}$ \\
\hline Rotation Speed & $0.16 \mathrm{sec} /$ 60degree (@7.4 Volt) \\
\hline Servo Accuracy & 0.24 degrees \\
\hline Torque & 17 Kg.cm (@7.4 Volt) \\
\hline Control Method & Serial Command (Baud rate - 115200bps) \\
\hline Parameter Feedback & Temperature, Voltage, Position \\
\hline
\end{tabular}

Table 1: Motor Parameters.

The control is made via serial communication through a single bus mode with a baud rate of 115200 bits per second. This communication uses a protocol based on custom command packets. Because of this way of communicating and controlling the motors, it is possible to connect several of them in series and control each one using their proper IDs in the command packet. This is extremely useful because only three pins (for the serial connection) are needed to control several motors.

\section{HAPTIC FORCE FEEDBACK}

The system is equipped with haptic force feedback. This is achieved using the servo motors that control the joints along with the load cells present in the remote robotic manipulator. The force values measured from the load cells of the remote arm are sent to the local arm's control software which then processes this information taking it into consideration when actuating the motors. In every control loop of the local arm, an updated value for the load cell's measure is calculated if the values taken from the remote load cells have different signs from the local ones. This essentially means that there are forces being applied in both arms opposing one another and therefore some sort of haptic feedback must take place. 
As a way to demonstrate this functionality, three plots will be displayed and analysed (one for each joint). In these plots there will be three series displayed: one for the position of the joint in the local arm, another for the force being applied on the local load cell to move the robotic manipulator and finally one for the force being applied on the remote load cell relative to that joint. The purpose of showing these three series is to demonstrate that when there is a force strong enough applied in the opposite direction of the movement, the joint does not continue moving even though the user keeps forcing the joint. This indicates that there is a constraint in that movement and therefore the user becomes more aware of the remote environment. Figures 18, 19 and 20 refer to joints 1, 2 and 3, respectively.

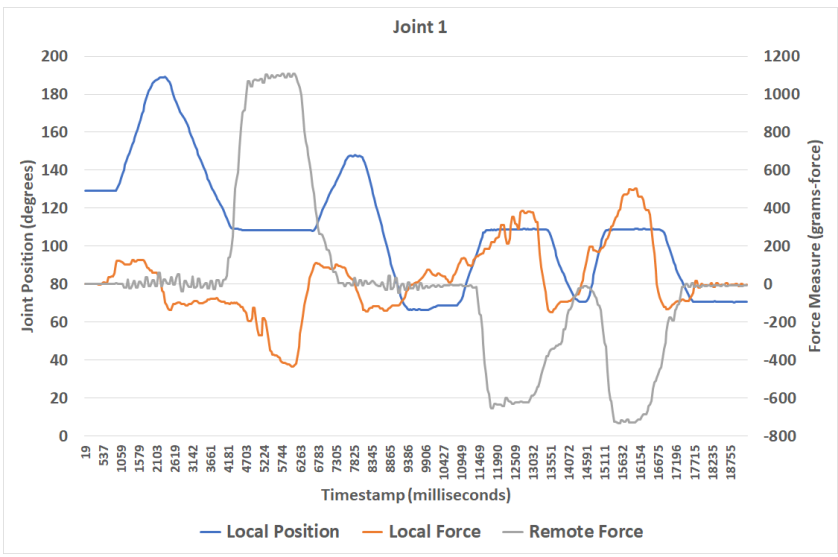

Figure 18: Joint 1 Haptic Feedback Plot.

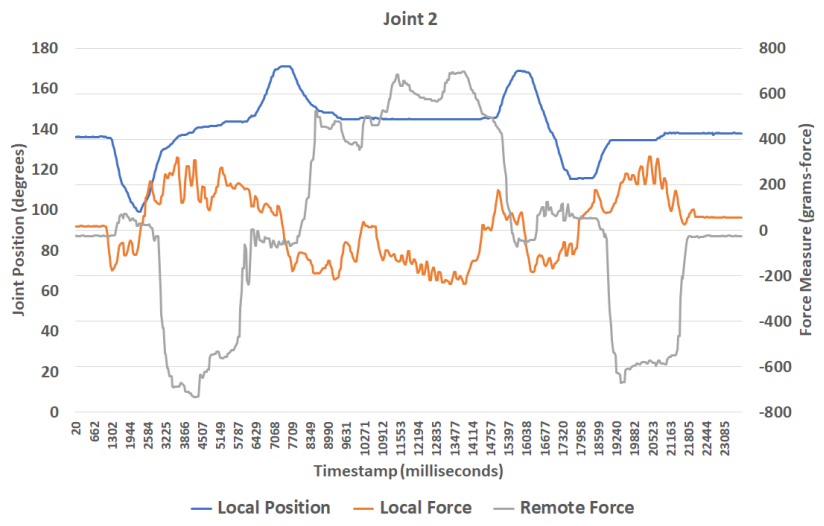

Figure 19: Joint 3 Haptic Feedback Plot.

Analyzing the first graph, Figure 18, which refers to the joint of the base, the first occurrence of haptic feedback can be seen at around 4200 milliseconds. At this time the remote force begins to rise will the joint is moving in the negative direction. This can be seen by the blue line, the joint position, descending as well as the orange line, the force being applied on that joint, being negative. As it is possible to see, the blue line stabilizes at around 110 degrees

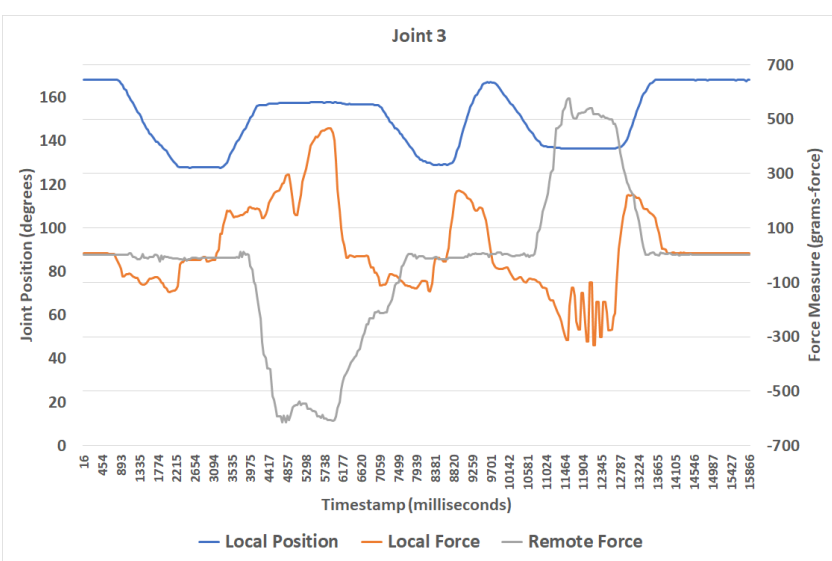

Figure 20: Joint 3 Haptic Feedback Plot.

even though the local force is strong enough for it to move in normal conditions. This reoccurs two more times at 11500 and 15100 milliseconds. The same thing happens for the remaining two joints. For the second joint, Figure 19, it happens at 2500, 8400 and 18600 milliseconds. Meanwhile, for the third joint, Figure 20 this occurs twice at 3900 and 11000 milliseconds.

\section{CONCLUSIONS AND FUTURE WORK}

This paper proposes a system intended for teaching remote robotic manipulators. The first steps of the development of the project were based around building the manipulator. The base model used was a palletizer configuration by the name EEZYbotArm MK2 which was provided by the user daGHIZmo on Thingiverse. After acquiring the base model of the manipulator to be used, began the project of the modifications, using Fusion 360, that needed to take place to convert the manipulator into it's desired form. This was an iterative procedure of modeling, printing, and validating the pieces projected. Once every piece had been projected and verified, what followed was building both manipulators with the servo motors, load cells and electromagnets. Having the manipulators ready for operation, the development of their control began, having as result a functional, low cost, modified robotic manipulator, that has a lot of potential, to be applied in control and robotics education. As future work the authors intend to explore the use of the proposed prototype in different educational contexts.

\section{ACKNOWLEDGMENTS}

This work has been supported by FCT - Fundação para a Ciência e Tecnologia within the Project Scope: UIDB/05757/2020.

\section{REFERENCES}

[1] Robert Bishop. 2002. Mechatronics Handbook (2nd. ed.). CRC Press, New York, NY.

[2] Jeremy D Brown, Andrew Paek, Mashaal Syed, M K O’Malley, P A Shewokis, J L Contreras-Vidal, and R. Brent Gillespie. 2013. Understandin[1] J. D. Brown, A. Paek, M. Syed, M. K. O'Malley, P. A. Shewokis, J. L. Contreras-Vidal, and R. B. Gillespie, "Understanding the Role of Haptic Feedback in a Teleoperated / Prosthetic Grasp and Lift Task," in Proc. IEEE World Haptics Conferen. In Proc. IEEE World Haptics Conference (2013-01-01). 271-276. 
[3] Miguel Á. Conde, Francisco J. Rodríguez-Sedano, Camino Fernández-Llamas, José Gonçalves, José Lima, and Francisco J. García-Peñalvo. 2021. Fostering STEAM through challenge-based learning, robotics, and physical devices: A systematic mapping literature review. Computer Applications in Engineering Education 29, 1 (2021), 46-65. https://doi.org/10.1002/cae.22354 arXiv:https://onlinelibrary.wiley.com/doi/pdf/10.1002/cae.22354

[4] Javier de Pedro, Gonzalo Esteban, Miguel Â. Conde, and Camino Fernández. 2016. HCore: a game engine independent $\mathrm{OO}$ architecture for fast development of haptic simulators for teaching/learning. In Proceedings of the Fourth International Conference on Technological Ecosystems for Enhancing Multiculturality, Salamanca, Spain, November 02 - 04, 2016, Francisco José García-Peñalvo (Ed.). ACM, 10111018. https://doi.org/10.1145/3012430.3012640

[5] Noguez J. Neri L. Escobar-Castillejos, D. 2016. Review of Simulators with Haptic Devices for Medical Training. J Med Syst. 40, 104 (2016). https://doi.org/10.1007/ s10916-016-0459-8
[6] Cristiana Ferrada-Ferrada. 2020. Robotics from stem areas in primary school: A systematic review. (2020)

[7] Carlo Franciscone. https://www.thingiverse.com/thing:1454048.

[8] Gowri Shankar Giri, Yaser Maddahi, and Kourosh Zareinia. 2021. An ApplicationBased Review of Haptics Technology. Robotics 10, 1 (2021). https://doi.org/10. 3390/robotics 10010029

[9] Juan González Martínez, Meritxell Estebanell Minguell, and Marta Peracaula Bosch. 2018. ¿Robots o programación? : el concepto de Pensamiento Computacional y los futuros maestros. (2018). https://doi.org/10.14201/ eks20181922945

[10] Hunter B. Gilbert Musa K. Jouaneh He Bai Kevin Stanley McFall, Kevin Huang and David M. Auslander. 2020. Mechatronics and Robotics Education: Standardizing Foundational Key Concepts. In 2020 ASEE Virtual Annual Conference Content Access. ASEE Conferences, Virtual On line. https://peer.asee.org/34966. 